\title{
Effects of Electropulsing Induced Microstructural Changes on THz-Reflection and Electrical Conductivity of Al-Doped Zno Thin-Films
}

\author{
Yaohua Zhu1 ${ }^{*}$, Weien Lai ${ }^{2}$ \\ ${ }^{1}$ Department of Industrial and Systems Engineering, Hong Kong Polytechnic University, Hong Kong, China \\ ${ }^{2}$ Faculty of Physics and Materials Sciences Center, Philipps-University Marburg, Marburg, Germany \\ Email: *yaohuazhu@hotmail.com
}

Received 3 May 2016; accepted 25 June 2016; published 28 June 2016

Copyright (C) 2016 by authors and Scientific Research Publishing Inc.

This work is licensed under the Creative Commons Attribution International License (CC BY). http://creativecommons.org/licenses/by/4.0/

\section{(c) (i) Open Access}

\begin{abstract}
Electropulsing induced phase transformation and crystal orientation change and their effects on electrical conductivity, THz reflection and surface roughness of thin-films of $\mathrm{Al}_{2} \mathrm{O}_{3}(2 \mathrm{wt} \%)$ doped ZnO were studied using XRD, SEM, AFM and Thz spectroscopy techniques. AZO-2 thin-films showed an effective response in $\mathrm{THz}$ spectroscopy under electropulsing. Electropulsing induced circular preferred crystal orientation changes and phase transformations were observed. The preferred crystal orientation changes accompanying decrease in stress and the secondary phase precipitation favored enhancing conductivity and THz reflection of the AZO-2 thin-films. After adequate electropulsing, both THz reflection and electrical conductivity of the thin-films were enhanced by $\mathbf{2 2 . 8 \%}$ and $\mathbf{6 . 8 \%}$, respectively; meanwhile surface roughness reduced. The property responses of electropulsing are discussed from point view of microstructural change and dislocation dynamics.
\end{abstract}

\section{Keywords}

Electropulsing, Precipitation, Preferred Crystal Orientation, THz Reflection, Electrical Conductivity, AZO Thin-Films

\section{Introduction}

Transparent Conductive Oxide (TCO) thin-films such as $\mathrm{In}_{2} \mathrm{O}_{3}, \mathrm{SnO}_{2}$ and $\mathrm{ZnO}$ have been widely studied for ${ }^{*}$ Corresponding author.

How to cite this paper: Zhu, Y.H. and Lai, W.E. (2016) Effects of Electropulsing Induced Microstructural Changes on THzReflection and Electrical Conductivity of Al-Doped ZnO Thin-Films. Journal of Surface Engineered Materials and Advanced Technology, 6, 106-117. http://dx.doi.org/10.4236/isemat.2016.63010 
their practical application as transparent electrodes, window in display, solar cells, and various optoelectronic devices [1]-[4]. Al-doped $\mathrm{ZnO}$ thin films have attracted much attention due to its easy in doping, high transmittance in visible region, high electrical properties and low cost [5] [6]. However, few studies have been carried out on microstructural evolution of the thin-films of semiconductor. Even less efforts have been made to improve these structural controlled properties, such as conductivity, and surface roughness.

In the past decades, the applications of thin-films of the functional semiconductors in the terahertz band received extensive attention [7]-[13]. Terahertz is a special period of the electromagnetic spectrum between microwave and infrared. The terahertz (THz) spectroscopy is becoming potential tools for characterizing or imaging various materials including semiconductor, high-temperature superconductor and biomaterial samples. $\mathrm{THz}$ has been known as the science and technology basis of the next generation of information industry in the international arena. However, unlike the microwave and optical bands, the natural materials lack of effective response to the $\mathrm{THz}$ band; the existing electronic devices and optical devices cannot achieve the $\mathrm{THz}$ transmission. The property responses of $\mathrm{THz}$ for the thin-films are still rudimentary.

As an advanced process, Electropulsing Treatment (EPT) has been proved to be a very effective process for improving the microstructures of materials and their properties [14]-[25]. Our previous work has shown that with adequate electropulsing, the elongation of the $\mathrm{Zn}-\mathrm{Al}$ based alloys was increased by 437\% [19]. Also, compared with the non-EPT thin-films of $\mathrm{Zn}-\mathrm{Al}$ alloy (ZA27) and thermoelectric $\left(\mathrm{Bi}_{0.25} \mathrm{Sb}_{0.75}\right)_{2} \mathrm{Te}_{3}$ semiconductor, electropulsing increases electrical conductivity by $18.2 \%$ and $28.6 \%$, respectively [20] [21]. It was reported that compared with that achieved in the conventional ageing, electropulsing tremendously accelerated phase transformations and microstructural changes by factors of at least 6000 and 3400 times for $\mathrm{Zn}-\mathrm{Al}$ and $\mathrm{Al}-\mathrm{Mg}$ alloys, respectively [22]-[24]. Circular phase transformations were detected in both bulk and thin-films of various alloys and the functional semiconductors [25]-[28]. Electropulsing offers an opportunity to explore a way in manufacturing thin-films of the semiconductors with appropriate properties required for the electronic devices.

In the present work, physical properties such as $\mathrm{THz}$ reflection, electrical conductivity and surface roughness of the thin-films of an Al-doped $\mathrm{ZnO}$ are studied by using electropulsing.

\section{Experimental Procedures}

Thin-films of an $\mathrm{Al}_{2} \mathrm{O}_{3}$ (2 wt\%) doped $\mathrm{ZnO}$ (AZO-2) were prepared by $\mathrm{RF}$ magnetron sputtering at room temperature $(300 \mathrm{~K})$. Sputtering was carried out at a pressure of $6 \times 10^{-3}$ Torr in pure argon gas with RF power 50 $\mathrm{W}$. The glass was used as the substrate. The glass-substrates coated with AZO-2 films of $1000 \mathrm{~nm}$ thickness were cut into pieces of $10 \mathrm{~mm}$ in width and $20 \mathrm{~mm}$ in length. Then we carried out electroulsing treatment (EPT) on the coated thin-films for various periods of time. The multiple positive electropules were applied. Operation parameters of pulse frequency, the root-mean-square value of current density were $1.6 \mathrm{KHz}$ and $20 \mathrm{~A} / \mathrm{mm}^{2}$, respectively. The EPT was carried out at room temperature (about $300 \mathrm{~K}$ ).

We did examination of the microstructural evolution and phase decomposition of the AZO-2 thin-film specimens after various periods of EPT by using X-diffraction (XRD), and scanning microscopy (SEM) techniques. XRD was performed using a X-ray diffractormeter (RIGAKU Smart Lab 9000W) with Ni-filed Cu $K_{\alpha}$-radiation $(\lambda=1.5418 \mathrm{~A})$ and scan-range was between $2 \theta=30^{\circ}$ and $60^{\circ}$. The scanning speed was 1 degree $/ \mathrm{min}$. Secondary electron images were collected, using a SEM Jeo-6490. Routing AFM examination was carried out using a Scanning Probe Microscope, Bruker Veeco Nanoscope V.

The electrical resistance of the thin film specimens was measured after various periods of EPT by a $R$-Check 4 point meter made by ELECTRIC DESIGN TO MARKET, INC USA. The results in ohms/square $\left(R_{\square}\right)$ were sheet resistance of the thin films. The sheet resistance can be written as

$$
R_{\square}=\frac{\rho}{d_{\text {film }}}
$$

where $\rho$ and $d_{\text {film }}$ is the resistivity and thickness of the AZO-2 film, respectively.

$$
\sigma=\frac{1}{\rho}
$$

where $\sigma$ is the conductivity of the AZO2 film.

From above two equations, the conductivity $\sigma$ of the $\mathrm{AZO} 2$ film is obtained as follows, 


$$
\sigma=\frac{1}{R_{\square} \times d_{\text {film }}} .
$$

Thus, relative increase of electrical conductivity of the EPT thin-films is obtained from the relative decrease of the sheet resistance in the present study [29].

AZO-2 thin-film specimens were also studied after various periods of EPT by using terahertz time-domain spectroscopy (THz-TDS) in a mode of reflection geometry at room temperature (300 K). Schematic diagram and operation parameters of the THz-TDS were illustrated previously [12]. A standard Au mirror assumed 100\% reflectivity was used as reference [13].

\section{Results and Discussion}

\subsection{Electropulsing Induce Microstructural Changes}

\subsubsection{Electropulsing Induced Phase Transformation}

The as coated AZO-2 thin-films consisted only one nanophase. Shown in Figure 1(a) are the XRD patterns of the supersaturates nanophases of the AZO-2 thin-films after EPT for 0, 3, 7, 15, 17 and 20 mins. Two characteristic XRD peaks of the supersaturated nanophase of hexagonal structure were selected as (0002) and $(10 \overline{1} 2)$ at $2 \theta=34.4$ and 47.5 degrees, respectively. It is seen that the nanophase was of a strong XRD at (0002) in the as coated thin-films. With increasing EPT time to 15 mins, the (0002) XRD decreased gradually, while the (10 $\overline{1} 2)$ increased. This meant that during 15 mins of EPT, The preferred crystal orientation changed from (0002) planes toward $(10 \overline{1} 2)$ planes, meanwhile precipitation of a secondary phase occurred. The XRD of the undetermined phase precipitate was found at $2 \theta=31.7$ degree. Upon further EPT, the $(10 \overline{1} 2)$ XRD gradually decreased, accompanying with increasing (0002) XRD. Meanwhile, the XRD of the Al-contained secondary precipitates (Alprecipitates) reduced in XRD intensity. A reverse transformation of the preferred crystal orientation and decomposition of the Al-precipitate occurred. This meant that, both circular preferred crystal orientation and the phase transformation occurred during 20 mins of electropulsing.

We carried out SEM examination after each XRD test. Shown in Figure 2(a) are SEM images of the AZO-2 thin-films after EPT for 0, 3, 7, 15 and 20 mins. It was found that clusters formed after EPT for 7 mins. After 15 mins of EPT, the clusters became Z-zones in the thin-films, shown in Figure 2(a). This formation of the Zones from the clusters of the nanophase was previously reported as in a continuous phase transformation [30]. After EPT for 15 mins, plenty of the Al-precipitate particles were observed of $85 \mathrm{~nm}$ in diameter, as shown in Figure 2(a). Upon further EPT for 20 mins, the Al-precipitates were found being degenerated and amount of the Alprecipitates reduced considerably. This indicated that the reverse phase transformation i.e. decomposition of the Al-precipitates occurred.

In agreement with XRD examination, the aforementioned the circular phase transformation was observed in SEM observation.

It was reported that during electropulsing, there was an impacting force of high-rate drift electrons as well as mass of collision between high-rate drift electrons and atomic nuclei [31]-[33]. Under the impact of transient stress, mobilized dislocations were moving very quickly, even at ultrasonic speeds [34].

It was proposed that the $\Delta \mathrm{G}_{\text {ep }}$ consisted normally of two parts; i.e. $\Delta \mathrm{G}_{\mathrm{ep}}=\Delta \mathrm{G}_{\text {therm }}+\Delta \mathrm{G}_{\text {atherm }}$, where $\Delta \mathrm{G}_{\text {therm }}$ is the Gibbs free energy resulting from Joule heat, i.e. the thermal effect. $\Delta G_{\text {atherm }}$ is the Gibbs free energy contributed by the athermal effect. It was estimated that the athermal effect of electropulsing was 319 times stronger than the thermal effect [35]. Thus, during electropulsing, the transfer energy directly from the electrons to the atoms was much more effective than that in the traditional thermal and thermo-mechanical processes.

The effect of heat treatment on crystal structure of AZO-2 thin-films was previously studied [36]-[38]. Neither the Al-precipitates, nor the distinct preferred crystal orientation at $(10 \overline{1} 2)$ planes (at $2 \theta=47.49$ degree $\mathrm{XRD}$ ) were observed during ageing at $673 \mathrm{~K}$ for 60 mins [36]. In comparison, both the circular phase transformations and the preferred crystal orientation changes were detected in the AZO-2 thin-films within 20 mins of electropulsing at the ambient temperature. Obviously, electropulsing tremendously accelerated phase transformation and microstructural changes in the AZO-2 thin-films.

\subsubsection{Electropulsing Induced Preferred Crystal Orientation Change}

One of the most important characteristics of the as coated thin-films of the TCO is the preferred crystal orientation. 


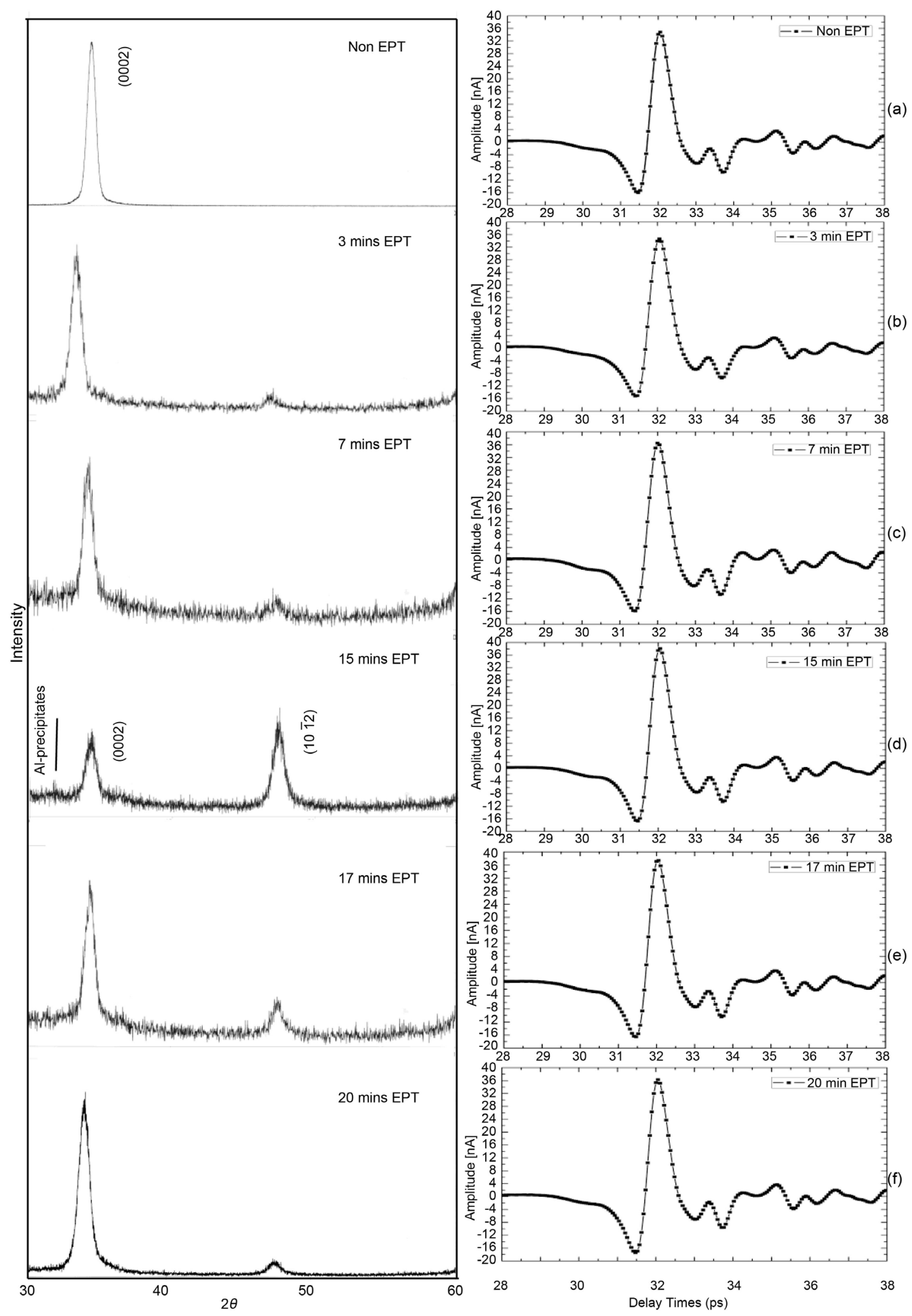

Figure 1. X-ray diffractograms (a) and THz refelction amplitude (b) of AZO-2 thin-films before (a) and after electropulsing for 3 (b), 7 (c), 15 (d), 17 (e) and 20 (f) mins, showing the second phase precipitates and the preferred crystal orientation at $(10 \overline{1} 2)$ favored enhancing the $\mathrm{THz}$ reflection. 


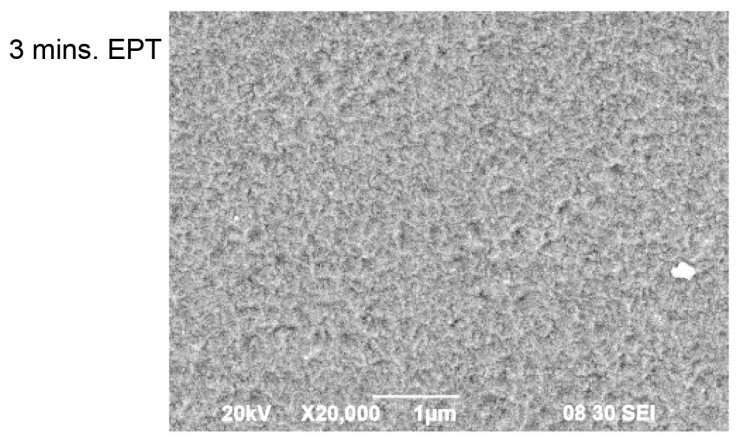

0 min. EPT

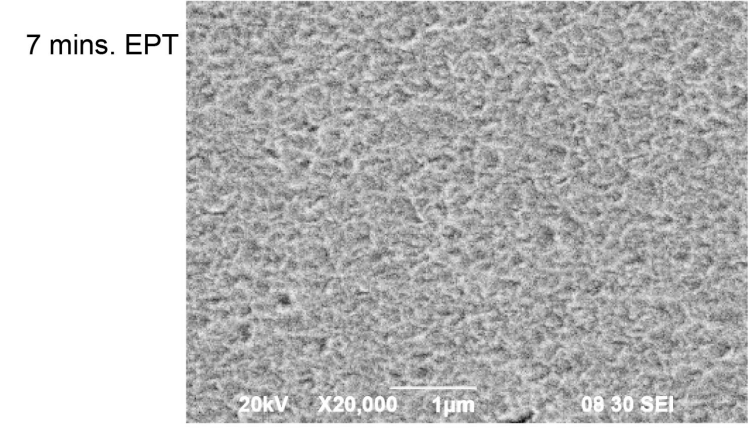

15 mins. EPT

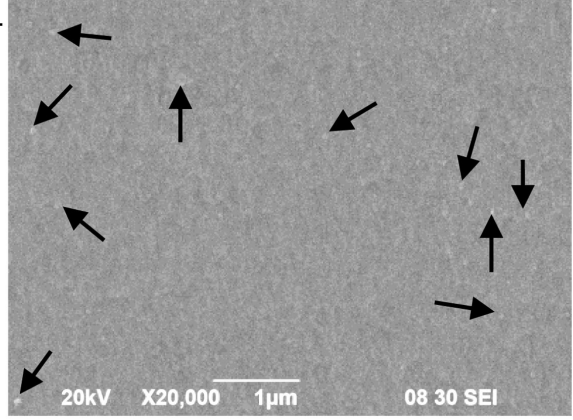

20 mins. EPT

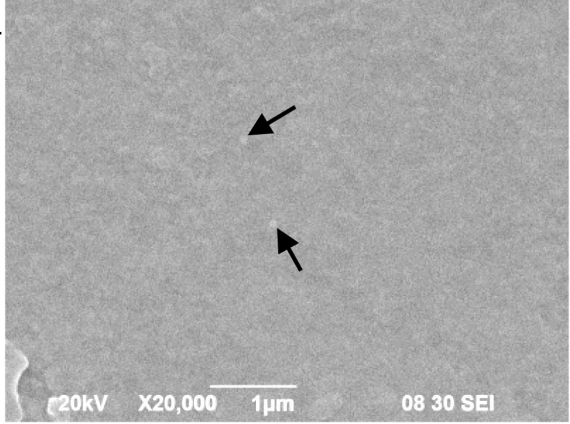

(a)
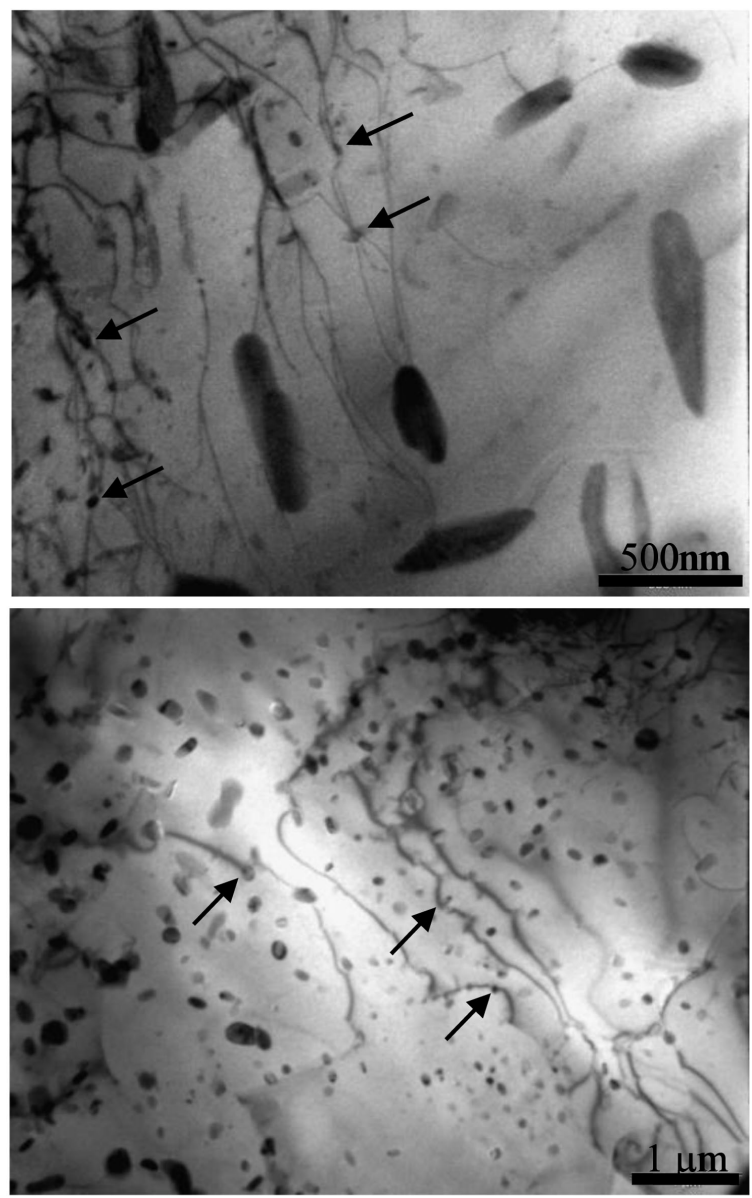

(b)

Figure 2. SEM images of AZO-2 thin-films after various periods of electropulsing (a) and typical TEM images of dislocation-pinning by discontinuous precipitates in EPT Zn-Al alloys (b), which favored enhancing carrier concentration.

It is important to study the co-relationship between the preferred crystal orientation and the stresses in the thinfilms.

The stress induced preferred crystal orientation changes in the tensile stress-deformed $\mathrm{Zn}$-Al alloy specimen (ZA22) were previously studied. The preferred orientation (10ī1) planes in the bulk part of the specimen is shown by a circle in Figure 3(a). Under the external stress, the preferred crystal orientation changes to (0002) planes in the rupture part of the tensile-deformed ZA alloy wire specimen, as indicated by a square in Figure $3(a)$. In the EBSD $\langle 11 \overline{2} 1\rangle$ inverse pole figure, the project-points concentrated at about (0001) corner in the 


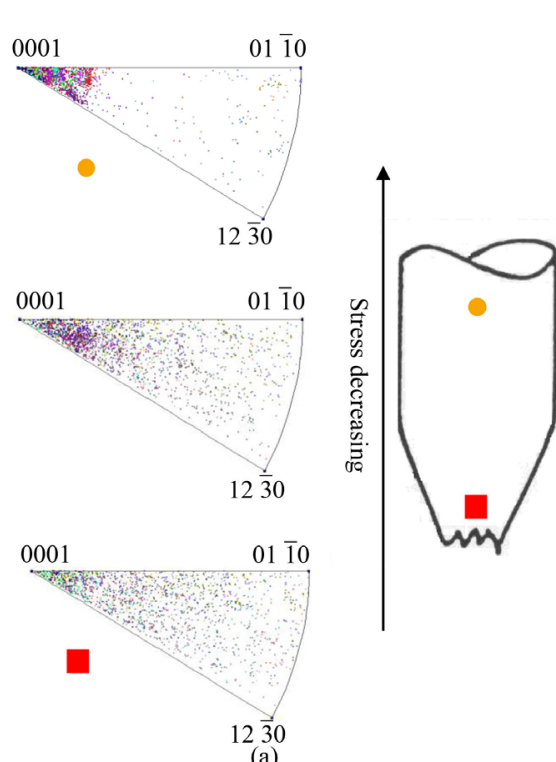

(a)

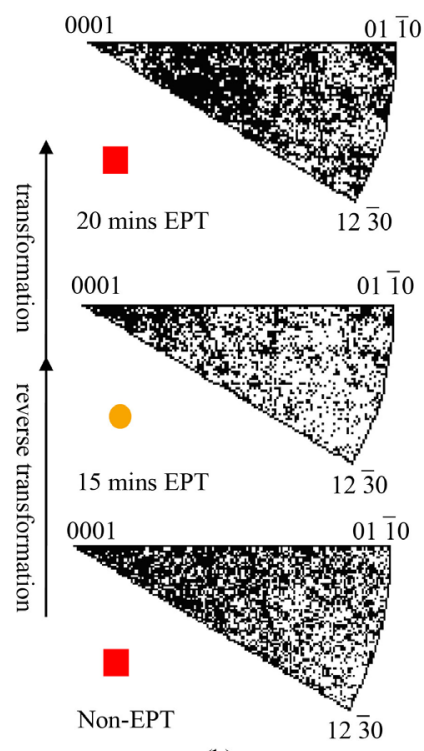

(b)

Figure 3. The $\langle 11 \overline{2} 1\rangle$ inverse pole figures of the $\eta^{\prime}$ т phase in the non-EPT tensile deformed (a) and the EPT cold rolled (b) ZA alloy wires with various current, showing the correlation between stress and the preferred crystal orientation.

bulk part of the specimen, and the project-points dispersed away from (0001) corner in the high-stressed rupture part of the specimen, as shown by a circle and a square in Figure 3(a) [37].

Also, the electropulsing induced preferred crystal orientation in cold deformed ZA22 wire specimen was studied, as shown in Figure 3(b). Under electropulsing, electron wind formed as an external stress, and drove dislocations to the grain boundaries and annihilated. The residue stress in the cold deformed specimens reduced. The EBSD project-points concentrated towards (0001) corner in the reverse pole figure, as shown by a circle in Figure 3(b). The preferred crystal orientation changed gradually from (0002) planes back to the original crystal orientation at $(10 \overline{1} 1)$ planes, accordingly the reverse phase transformation occurred.

Upon further electropulsing, the external stress induced preferred crystal orientation changes were observed, and the EBSD project-points dispersed again away from (0001) corner in the EBSD $\langle 11 \overline{2} 1\rangle$ inverse pole figure, as shown by a square in Figure 3(b). Because of the increasing external stress, the preferred crystal orientation changed to the high-stressed (0002) planes from the $(10 \overline{1} 1)$ planes [26]. The destroying the high stress crystal orientation, i.e. contrast in stress was shown quantitatively by EBSD results in Figure 3 [39] [40].

In the present study, because of the rapid solidification the nanophase possessed a strong preferred crystal orientation at (0002) planes in the as coated AZO-2 thin-films. Under electropulsing, the preferred orientation destroyed and changed from the high-stressed (0002) planes of the as coated state back to (10 $\overline{1} 2)$ planes, while the stress reduced to minimum, as shown by a circle in Figure 2. Upon further electopulsing, electron wind appeared as an external stress, forcing the crystal orientation away from the original orientation $(10 \overline{1} 2)$ planes to the high-stressed (0002) planes, accompanying the reverse phase transformation i.e. the decomposition of the Al-precipitates, as shown in Figure 2. The aforementioned preferred crystal orientation from the highstressed state to the low-stressed state and the reverse were observed in the thin-films of both the ZA alloys and the $\left(\mathrm{Bi}_{0.25} \mathrm{Sb}_{0.75}\right)_{2} \mathrm{Te}_{3}$ semiconductor [20] [21].

It is reasonable to point out that the preferred crystal orientation was closely related with stresses in the AZO-2 thin films.

\subsection{Electrical Conductivity of the AZO-2 Thin-Films}

The electrical conductivity of the AZO-2 thin-films was deduced from the measured sheet resistance, $R_{\square}$, which were plotted against various periods of electropulsing $(0,1,3,7,15$, and 20 mins), as shown in Figure 4(a).

High electrical conductivity $(\sigma)$ required a large electron charge $e$, a high carrier concentration $n$ and a large mobility $\mu$, through $\sigma=n e \mu$. Under electropulsing, achieving the balance became much more complicated. 

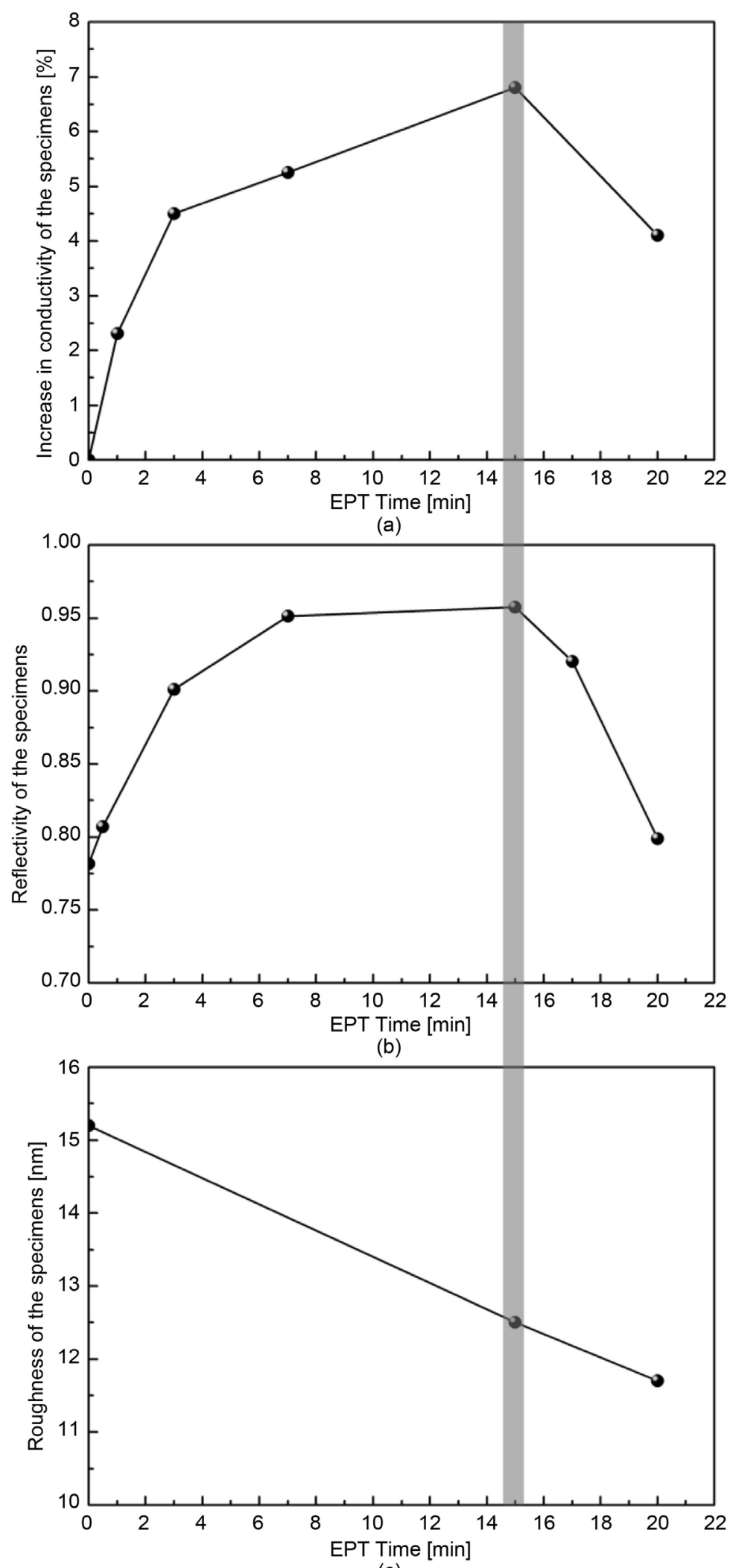

(c)

Figure 4. Relative increases of electrical conductivity (a), THZ reflectivity (b) and surface roughness (c) of AZO-2 thin-films after various periods of electropulsing. 
During electropulsing, the accumulation and annihilation of dislocation occurred at grain boundaries and defects. At early stage of elcetropulsing, the strong preferred crystal orientation was destroyed [20]. The dislocation increased greatly, and the carrier concentration increased accordingly [20] [21]. Meanwhile, the residue stress in the as coated thin-films considerably decreased. The interaction between electrons and the stress field became week. As a result, the conductivity enhanced by at a high rate. Within first 3 mins. of EPT, the conductivity increased by $4.5 \%$. With increasing time of EPT, the dislocation density reduced through annihilation of dislocation at grain boundaries. Accordingly, the carrier concentration decreased and the increase rate of the conductivity reduced. After EPT for 7 mins, the conductivity increased by 5.3\%, i.e. within the following 4 mins the conductivity increased only for $1.2 \%$, as shown Figure 4(a).

The electropulsing induced phase transformation influenced the dislocation dynamics. After EPT for 15 mins, the secondary phase Al-precipitates well developed, as shown in Figure 1 and Figure 2. The dislocations were pinned by the electropulsing induced the precipitates, which increased density of the dislocation. A typical TEM images of dislocations pinning by discontinuous precipitates in EPT ZA alloys were previously presented [21]. As a result, the carrier concentration and vacancies increased [21], and the electrical conductivity enhanced by 6.8\% to the maximum after EPT for 15 mins. Upon further electropulsing, the reverse phase transformation occurred and the Al-precipitates gradually degenerated. Meanwhile the dislocation annihilation was accelerated. Thus, electrical conductivity decreased quickly. After EPT for 20 mins., the conductivity increased 4.1\%, compared with that of the non-EPT thin-films. It can be seen that destroying the high-stressed crystal orientation at (0002) planes played an important role in enhancing the conductivity.

\subsection{THz Reflection Responses of EPT AZO-2 Thin-Films}

The THz Time domain waveforms, i.e. THz reflection amplitude vs delay time, of AZO thin-films are shown in Figure 1(b). At early stage of electropulsing, the $\mathrm{THz}$ reflection rapidly increased with increasing time, and reached the maximum after electropulsing for 15 mins. The THz reflection increased by a factor of $22.8 \%$, compared that of the as coated AZO-2 thin-films. Then THz reflection decreased during the prolonged electropulsing, as shown in Figure 1(b).

The AZO-2 thin-films were of very effective THz responses within a wide range of THz band (0.1 - $1.6 \mathrm{THz})$. After EPT for 15 mins, the terahertz waveforms of AZO films were very close to that of the standard Au mirror in the time domain, being over $96 \%$ of Au standard sample (100\%), as indicated by a solid line in Figure 5(a). Outside this frequency range, the signal was too weak to obtain an acceptable signal-to-noise ratio in our terahertz system.

Theoretical (gray) and experimental (dark) reflection amplitude of AZO-2 thin-films are shown in Figure 5(b). It can be seen that the experimental results from reflection measurements were in a good agreement with that predicted by the theoretical Drude-model. The details of the theoretical calculation of Drude model were previously described [12].

Within the THz frequency bands, the electromagnetic wave interacted with free carrier, and scattered within unit cells or defects such as dislocations, boundaries and voids etc., which would reflected the THz phonons. Thus, the $\mathrm{THz}$ reflection was closely related to the microstructural defects, i.e. the carrier concentration in the AZO-2 thin-films.

The electropulsing induced THz reflection changes followed a similar circulation to that the electrical conductivity underwent. At the early stage of EPT, the significant increase in dislocation density and carrier concentration because of destroying the high-stressed preferred crystal orientation at the (0002) planes, and the $\mathrm{THz}$ reflection increased greatly. After EPT for 15 mins, the Al-precipitates considerably increased, accordingly the dislocation density and the carried concentration increased to the maximum. Accordingly, both the highest electrical conductivity and the strongest $\mathrm{THz}$ reflection were observed.

When electromagnetic wave arrived at the AZO-2 thin-films, reflection, transmission and absorption occurred. When the transmission remained unchanging, the reflection was inversely proportional to the absorption. When an external energy field such as stress field or electric-magnetic field existed, the scattering of the THz phonons was affected, and part of the THz microwave energy was absorbed through interacting with the external energy field. At early stage of EPT, destroying the high-stressed crystal orientation at the (0002) planes reduced the interaction. The THz reflection dramatically enhanced. After EPT for 15 mins, the stress reduced to the minimum, the $\mathrm{THz}$ reflection increased to the maximum. Within the first 3 mins of Thz reflection, the THz reflection increased by $15.3 \%$. Destroying the high-stressed crystal orientation at (0002) planes played an important role in enhancing the $\mathrm{THz}$ reflection. 


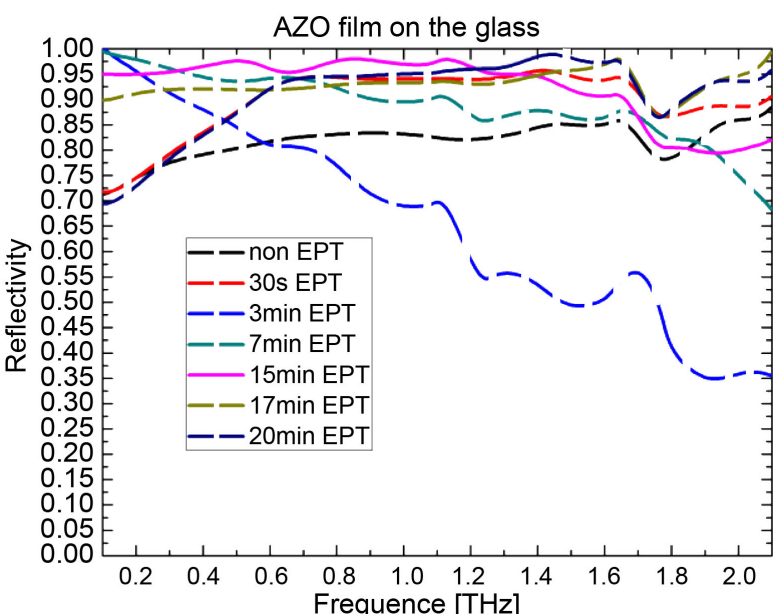

(a)

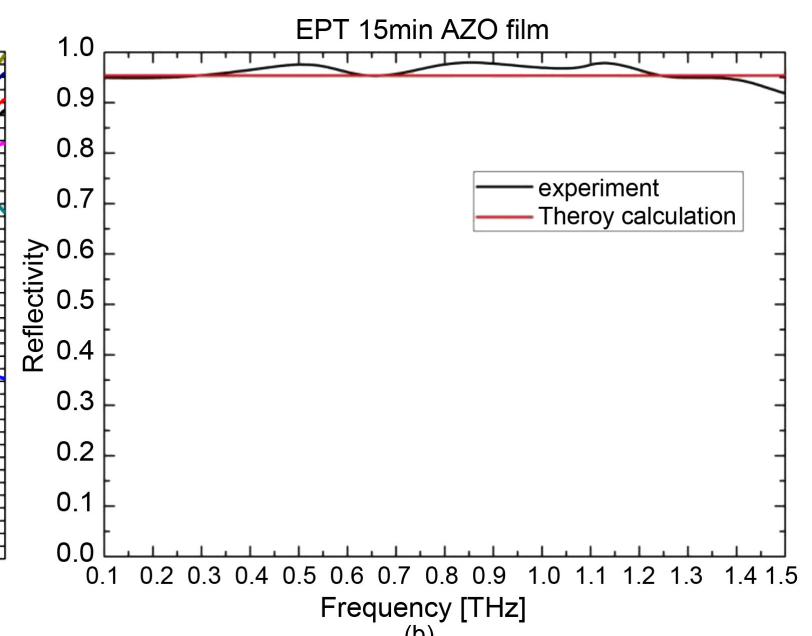

(b)

Figure 5. THz reflection amplitude vs delay time (a) and theoretical (gray) and experimental (dark) amplitude reflectivities (b) of the AZO-2 thin-films.

Upon further electropulsing, the THz reflection reduced after EPT for 20 mins. The decrease in THz reflection was attributable to the decrease in the discontinuous secondary Al-precipitates and increase in the stress in the thin-films.

From the aforementioned discussion, it can be seen that both the discontinuous secondary precipitation and the preferred crystal orientation changes accompanying decrease in stress favored enhancing both electrical conductivity and THz reflection in the AZO-2 thin-films, and vice versa. Destroying the high-stressed crystal orientation played an important role in enhancing the properties.

\subsection{Roughness of EPT the Thin-Films}

In the supersaturated solid phase, grain grew as pyramids along with the strong preferred crystal orientation at (0002) planes, as shown in Figure 2(a) and Figure 6(a). The surface roughness of the thin-films was the room mean square value (RMS) $R_{q}=15.2$. After EPT for 7 mins, nanophase had started to cluster and form zones, shown in Figure 2(a). The zones were well developed after EPT for 15 and 20 mins. The roughness was reduced to $R_{q}=12.5$ and 11.7, as shown in Figure 6(b) and Figure 6(c) respectively.

Decomposition of nanophase consisted of two parts of discontinuous and continuous. It was reported that the supersaturated nanophase possessed a very strong preferred crystal orientation. At the early stage of ageing, decomposition of the nanophase was observed to start with clustering to form zones, transitional phases and metastable phases. The decomposition of the nanophase was tremendously retarded because of the strong preferred crystal orientation [30]. In the present study, the continuous decomposition of the nanophase was observed after EPT for 7 mines. Formation of the zones developed after EPTs for 15 and 20 mins, respectively, accordingly, the roughness reduced to $R_{q}=12.5$ and 11.7, respectively. Meanwhile the discontinuous precipitation was developed after EPT for 15 mins. Obviously the formation of zones favored reducing the roughness of the AZO-2 thin-films. It is interested to notice that after EPT for 15 mins, roughness reduced meanwhile both the electrical conductivity and the THz reflection reached the highest values.

\section{Conclusions}

From the above reported studies, the conclusions were made as follows:

1) Electropulsing tremendously accelerated phase transformation and microstructural changes in the AZO-2 thin-films. Electropulsing induced circular transformation and the preferred crystal orientation changes were observed within 20 mins. in the AZO-2 thin-films.

2) The preferred crystal orientation was closely related with stresses in the AZO-2 thin-films. The electropulsing induced preferred crystal orientation changes from high-stressed state to low-stressed state, and the reverse, were closely related with physical property changes. 

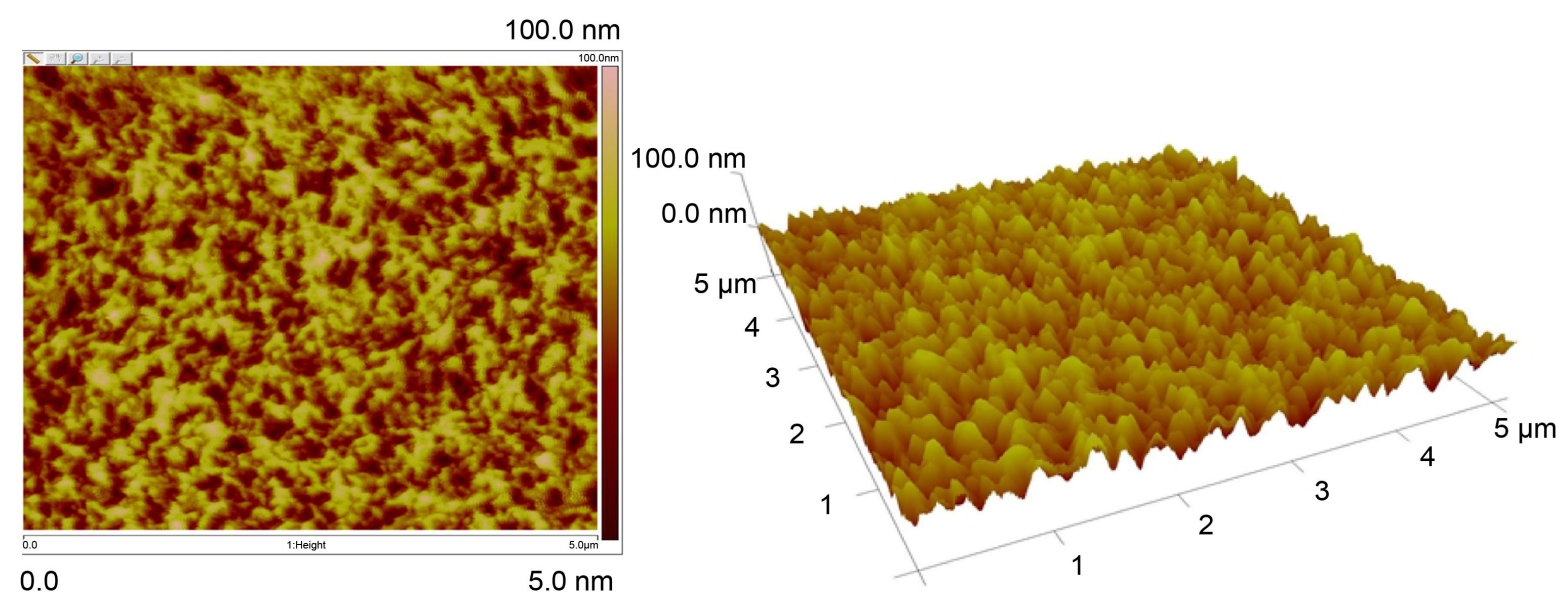

(a)
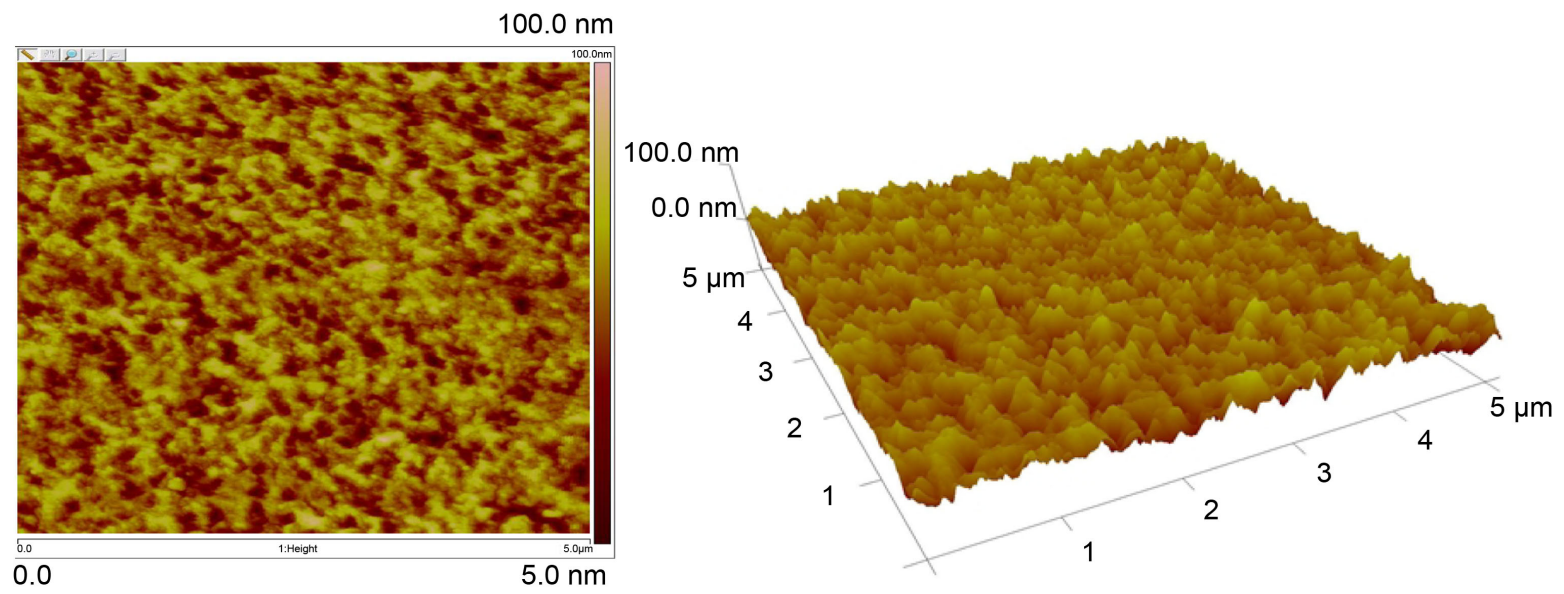

(b)
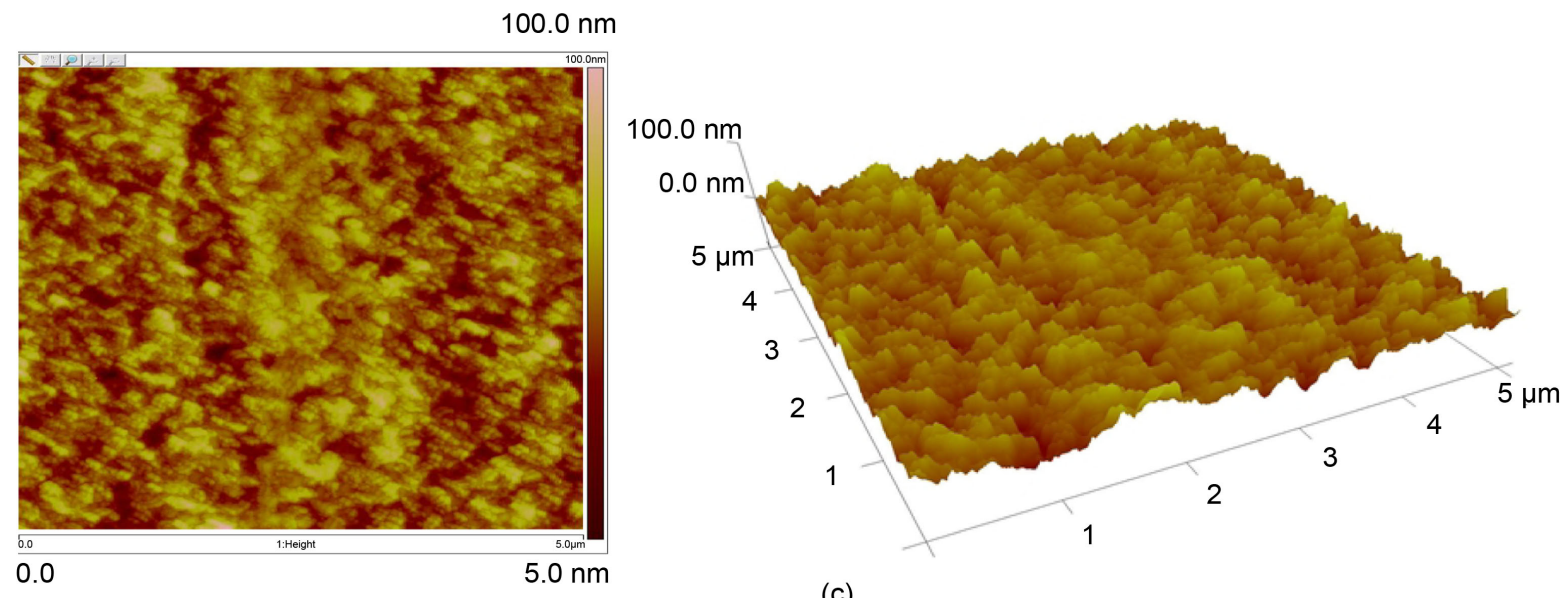

(c)

Figure 6. AFM images of the AZO-2 thin-films after EPT for 0 (a), 15 (b) and 20 (c) mins.

3) Both the discontinuous secondary precipitation and the preferred crystal orientation changes accompanying decrease in stress favored enhancing both electrical conductivity and THz reflection in the AZO-2 thin-films, and vice versa. Destroying the high-stressed crystal orientation at the (0002) planes played an important role in enhancing the properties.

4) After adequate electropulsing, both the electrical conductivity and the $\mathrm{THz}$ reflection increased, meanwhile the roughness of the AZO-2 thin-films reduced considerably. After EPT for 15 mins, both the THz reflection and 
the electrical conductivity increased by $22.8 \%$ and $6.4 \%$, respectively. Whilst the roughness reduced to $R_{q}=12.5$.

5) AZO-2 thin-films were of very effective $\mathrm{THz}$ responses within a wide range of $\mathrm{THz}$ band $(0.1-1.5 \mathrm{THz})$, reflection of the $\mathrm{THz}$ reached over $96 \%$ of Au standard sample after an adequate electropulsing.

\section{Acknowledgements}

Authors would like to express their thanks to Mr. Jeremy Yeung of Materials Research Center of Hong Kong Polytechnic University for his assistances in Microscopy lab work.

\section{References}

[1] Parthiban, S., et al. (2010) Investigations on High Visible to Near Infrared Transparent and High Mobility Mo Doped $\mathrm{In}_{2} \mathrm{O}_{3}$ Thin Films Prepared by Spray Pyrolysis Technique. Solar Energy Materials and Solar Cells, 94, 406-412. http://dx.doi.org/10.1016/j.solmat.2009.10.017

[2] Kelmer, H. (2012) Past Achievements and Future Challenges in Development of Optically Transparent Electrodes. Nature Phontonics, 6, 808-816.

[3] Fortunato, E. (2012) Oxide Semiconductor Thin-Film Transistor: A Review of Recent Advances. Advanced Materials, 24, 2945-2986. http://dx.doi.org/10.1002/adma.201103228

[4] Castaneda, L. (2011) Present Status of Development and Application of Transparent Conductors Oxide Thin Solid Films. Materials Sciences and Applications, 2, 1233-1242. http://dx.doi.org/10.4236/msa.2011.29167

[5] Afluga, P., Sittingera, V., Ruskea, F., Szyszkaa, B. and Dittmarb, G. (2004) Optical Characterization of AluminumDoped Zinc Oxide Films by Advanced Dispersion Theories. Thin Solid Films, 455-456, 201-206. http://dx.doi.org/10.1016/j.tsf.2004.01.006

[6] Hiramatsu, M., Kmaeda, I., Horio, N. and Nawata, M. (1998) Trasparent Conducting ZnO Thin Films Prepared by XeCl Excimer Laser Ablation. Journal of Vacuum Science \& Technology A, 16, 669-673. http://dx.doi.org/10.1116/1.581085

[7] Nahata, A., Auston, D.H., Heinz, T.F. and Wu, C. (1996) Coherent Detection of Freely Propagating Terahertz Radiation by Electro-Optic Sampling. Applied Physics Letters, 68, 150-152. http://dx.doi.org/10.1063/1.116130

[8] Hangyo, M., Nagashima, T. and Nashima, S. (2002) Spectroscopy by Pulsed Terahertz Radiation. Measurement Science and Technology, 13, 1727-1738. http://dx.doi.org/10.1088/0957-0233/13/11/309

[9] Ferguson, B., et al. (2002) Materials for Terahertz Science and Technology. Nature Materials, 1, 6-33. http://dx.doi.org/10.1038/nmat708

[10] Beard, M.C., Turner, G.M. and Schmuttenmaer, C.A. (2002) Terahertz Spectroscopy. Journal of Physical Chemistry B, 106, 7146-7159. http://dx.doi.org/10.1021/jp020579i

[11] Brunner, F.D.J., Schneider, A. and Gunter, P. (2009) A Terahertz Time-Domain Spectrometer for Simultaneous Transmission and Reflection Measurements at Normal Incidence. Optics Express, 17, 20684-20693. http://dx.doi.org/10.1364/OE.17.020684

[12] Lai, W.E., Zhang, H.W., Zhu, Y.H. and Wen, Q.Y. (2013) A Novel Method of Terahertz Spectroscopy and Imaging in Reflection Geometry. Applied Spectroscopy, 67, 36-39. http://dx.doi.org/10.1366/12-06713

[13] Khazan, M., Meissner, R. and Ingrid, W. (2001) Convertible Transmission Reflection Time-Domain Terahertz Spectrometer. Review of Scientific Instruments, 72, 8. http://dx.doi.org/10.1063/1.1384433

[14] Troitskii, O.A. (1969) Electromechnical Effect in Metals. Zh. Eksp. Teor. Fiz, 10, 18-22.

[15] Gromov, V.E., Zuev, L.B., Bazaikin, V.I. and Tsellermaer, V.Y. (1996) Laws of Electrical Stimulation of Plastic Development of Metals and Alloys at Various Structural Levels. Russian Physics Journal, 39, 237-261. http://dx.doi.org/10.1007/BF02067645

[16] Qin, R.S. and Zhou, B.L. (1998) Effect of Electric Current Pulses on Grain Size in Castings (Overview). International Journal of Non-Equilibrium Processing, 11, 77-86.

[17] Stolyarov, V.V. (2009) Deformability and Nanostructuring of TiNi Shape-Memory Alloys during Electroplastic Rolling. Materials Science and Engineering: A, 503, 18-20. http://dx.doi.org/10.1016/j.msea.2008.01.094

[18] Conrad, H. (2000) Effect of Electric Current on Solid-State Phase Transformations in Metals. Materials Science and Engineering A, 287, 227-237.

[19] Zhu, Y.H., Lee, W.B, To, S., Liu, X.M., Jiang, Y.B. and Tang, G.Y. (2009) Effect of Dynamic Electropulsing on Microstructure and Elongation of a Zn-Al Based Alloy. Materials Science and Engineering: A, 501, 125-132. http://dx.doi.org/10.1016/j.msea.2008.09.080 
[20] Zhu, Y.H., Jiang, S.Q., Zhao, D.D., Cheng, G.J., Zhang, H.W. and Lai, W.E. (2013) Effects of Electropulsing on Microstructure and Conductivity of Nanofilms of Zn-Al Alloys. Applied Physics A, 111, 1241-1245. http://dx.doi.org/10.1007/s00339-012-7396-2

[21] Zhu, Y.H., Jiang, J., Xiao, Y.K., Luk, C.M. and Lai, W.E. (2013) Electropulsing Induced Microstructure Evolution and Its Effect on Electrical Conductivity of $\left(\mathrm{Bi}_{0.25} \mathrm{Sb}_{0.75}\right)_{2} \mathrm{Te}_{3}$ Thin Films. Scripta Materialia, 69, 219-222. http://dx.doi.org/10.1016/j.scriptamat.2013.03.032

[22] Zhu, Y.H., To, S., Lee, W.B., Liu, X.M., Jiang, Y.B. and Tang, G.Y. (2009) Electropulsing Induced Phase Transformation in a Zn-Al Based Alloy. Journal of Materials Research, 24, 2661-2669. http://dx.doi.org/10.1557/jmr.2009.0300

[23] Zhang, D., To, S., Zhu, Y.H., Wang, H. and Tang, G.Y. (2012) Static Electropulsing Induced Microstrcutural Changes and Their Effects on Ultra-Precision Machining of a Cold-Rolled Alloy AZ91. Metallurgical and Materials Transactions A, 43, 1341-1346. http://dx.doi.org/10.1007/s11661-011-0955-X

[24] Jiang, Y.B., Tang, G.Y., Shek, C.H., Zhu, Y.H. and Xu, Z.H. (2009) On the Thermodynamics and Kinetics of Electropulsing Induced Dissolution of $\beta-\mathrm{Mg}_{17} \mathrm{Al}_{12}$ Phase in an Aged Mg-9Al-1Zn Alloy. Acta Materialia, 57, 4797-4808. http://dx.doi.org/10.1016/j.actamat.2009.06.044

[25] Hu, G.L., Zhu, Y.H., Shek, C.H. and Tang, G.Y. (2011) Electropulsing-Induced G-Texture Evolution in a Deformed Fe-3\%Si Alloy Strip. Journal of Materials Research, 26, 917-922. http://dx.doi.org/10.1557/jmr.2010.101

[26] Zhu, Y.H., To, S., Liu, X.M., Jiang, Y.B. and Hu, G.L. (2011) Effect of Static Electropulsing on Microstructure and Elongation of a Zn-Al Alloy (ZA22). Metallurgical and Materials Transactions A, 42, 1933-1940. http://dx.doi.org/10.1007/s11661-010-0591-X

[27] Zhu, Y.H., To, S., Liu, X.M., Hu, G.L. and Xu, Q. (2011) Static Electropulsing Induced Phase Transformations in Cold Deformed ZA27 Alloy. Journal of Materials Research, 26, 1696-1701. http://dx.doi.org/10.1557/jmr.2011.185

[28] Zhu, Y.H., To, S. and Liu, X.M. (2012) Influence of Spinodal Decomposition on Plastic Behavior of Dynamic Electropulsing Treated ZA22 Alloy. Materials Transactions, 53, 1363-1370. http://dx.doi.org/10.2320/matertrans.M2012024

[29] Jaeger, R.C. (2001) Introduction to Microelectronic Fabrication. Prentice Hall, Upper Saddle River, 81-88.

[30] Zhu, Y.H., Chung, C.Y. and Lee, W.B. (2004) Nanophase Decomposition in Eutectoid Zn-Al Based Alloy Films. Materials Science and Engineering: A, 374, 145-152. http://dx.doi.org/10.1016/j.msea.2004.01.038

[31] Conrad, H. and Sprencher, A.F. (2009) The Electroplastic Effect in Metals. Dislocation in Solid. Elsvier, Amstterdam, 497.

[32] Sprecher, A.F., Mamnna, S.L. and Conrad, H. (1986) On the Mechanisms for the Electroplastic Effect in Metals. Acta Metallurgica, 34, 1145-1162. http://dx.doi.org/10.1016/0001-6160(86)90001-5

[33] Hao, T., Tanimoto, H. and Mizubayashi, H. (2005) Transformation to Nanocrystallites in Amorphous Alloys Induced by Resonant Electropulsing. Materials Transactions, 46, 2898-2930. http://dx.doi.org/10.2320/matertrans.46.2898

[34] Gumbsch, P. and Gao, H.J. (1998) Dislocations Faster than the Speed of Sound. Science, 283, 965-968. http://dx.doi.org/10.1126/science.283.5404.965

[35] Hu, G.L., Tang, G.Y., Zhu, Y.H. and Shek, C.H. (2011) Electropulsing Induced Texture Evolution in the Recrystallization of Fe-3\%Si Alloy Strip. Metallurgical and Materials Transactions A, 42, 3484-3490. http://dx.doi.org/10.1007/s11661-011-0770-4

[36] Miao, D.G., Jiang, S.X., Jiang, S.M. and Chen, Z.M. (2014) Effect of Heat Treatment on Infrared Property of Al Doped ZnO Films. Solar Energy Materials \& Solar Cells, 127, 163-168. http://dx.doi.org/10.1016/j.solmat.2014.04.030

[37] Kuo, S., et al. (2006) Effects of Dopping Concentration and Annealing Temperature on Properties of Highly-Oriented Al-Doped ZnO Films. Journal of Crystal Growth, 287, 78-84. http://dx.doi.org/10.1016/j.jcrysgro.2005.10.047

[38] Wimmer, M., Ruske, F., Scherf, S. and Rech, B. (2012) Improving the Electrical and Optical Properties of DC-Sputtered ZnO:Al by Thermal Post Deposition Treatment. Thin Solid Films, 520, 4203-4207. http://dx.doi.org/10.1016/j.tsf.2011.04.102

[39] Zhu, Y.H. (2004) General Rule of Phase Decomposition of Zn-Al Based Alloys (II)—On Effects of External Stress on Phase Transformation (Overview). Materials Transactions, 45, 3083-3097. http://dx.doi.org/10.2320/matertrans.45.3083

[40] Zhu, Y.H., Lee, W.B. and To, S. (2003) Use of EBSD to Study Stress Induced Microstructural Changes in Zn-Al Based Alloy. Materials Science and Engineering: A, 348, 6-14. http://dx.doi.org/10.1016/S0921-5093(02)00252-6 


\section{Submit or recommend next manuscript to SCIRP and we will provide best service for you:}

Accepting pre-submission inquiries through Email, Facebook, Linkedin, Twitter, etc A wide selection of journals (inclusive of 9 subjects, more than 200 journals)

Providing a 24-hour high-quality service

User-friendly online submission system

Fair and swift peer-review system

Efficient typesetting and proofreading procedure

Display of the result of downloads and visits, as well as the number of cited articles

Maximum dissemination of your research work

Submit your manuscript at: http://papersubmission.scirp.org/ 